\title{
Refractory Primary Cutaneous Lymphoma
}

National Cancer Institute

\section{Source}

National Cancer Institute. Refractory Primary Cutaneous Lymphoma. NCI Thesaurus.

Code C142877.

Primary cutaneous lymphoma that does not respond to treatment. 